\title{
Multi - channel Electrical Information Acquisition and Control System based on PLC and RS485 Bus Network
}

\author{
Zhengyu Xie, Cai Wen, Yansong Deng * \\ Institute of Electrical and Information Engineering, Southwest University for Nationalities of China, \\ Chengdu, Sichuan, 610041, P.R. China \\ *Corresponding author: Email:342547288@qq.com
}

Keywords: PLC, RS485 Bus Network, Isolated Hall current sensor.

\begin{abstract}
Based on PLC control technology and RS485 bus network communication technology, a multi - channel electrical information acquisition and control system provided by this paper for teaching building to achieve centralized management of the entire teaching building and prevent serious waste of energy resources. The whole system is based on pc host computer, the plc controller for the slave, through the RS485 bus network communication, to achieve the real-time feedback control between a number of slave and a host. The system can be used to achieve the statistics of realtime power consumption for classroom, and provide energy-saving with decision-making basis, with a wide range of applications.
\end{abstract}

\section{Introduction}

At present, in the classroom resource open management mode, due to the lack of corresponding efficient deployment management automation system, many classroom energy waste is very serious, often one or two personal, and even in nobody case, classroom lighting, fans and other long-term running in the state. To better solve the issues, this paper applies a building Management System based on PLC and 485 bus network. Managers can view and control the power consumption of any classroom directly through the man-machine interaction interface. This system uses the Hall current transformer and the ad circuit that integrated on plc to form a feedback network to learn that the electrical load, which can detect the subtle changes in power consumption in real time, so the sensitivity is high. Rs485 is used as the main transmission line, with the data needle address detection means, can achieve a non-interference, safe and reliable control system. Using the corresponding address strategy let the different plc controller assigned to a different address, greatly optimized the performance of data transmission recognition, and the reliability of the equipment to enhance. The administrator can customize the changes and time limit the load of electricity units according to the unit electrical load displayed on the PC. The system not only saves resources, promptly detects faults, but also facilitates the use of electricity statistics, with a very good practical value.

\section{System composition}

Aiming at the problems of low level of informatization, low level of automation, insufficient resource utilization and serious energy waste in classroom management of classroom resources in colleges and universities, the advanced control technology is used to construct the energy saving control and management system of classroom resources based on RS485 network bus. The composition of multi - channel electric information collection and control system based on PLC and RS485 bus network includes the classroom terminal controller PLC, the terminal acquisition unit, RS485 bus network, the unified management software for centralized control and monitoring and other components. The whole system through the differential 485 bus network and USB-484 protocol conversion network to interconnect the various parts organically and form a bus-type data communication network. The specific network connection diagram shown in Figure 1: 


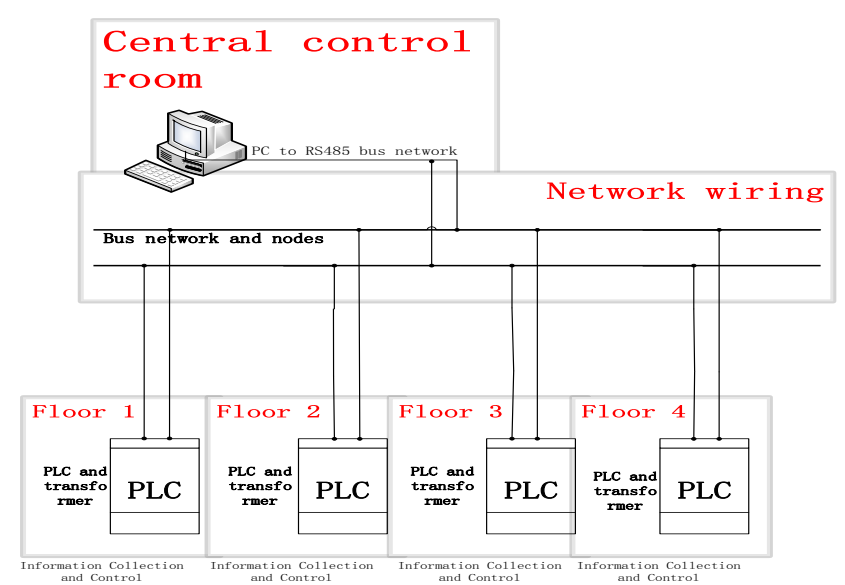

Figure 1. System network connection diagram

\section{Terminal controller PLC}

In view of the classroom generally use a floor of each distribution box design, so the system control and power acquisition design in the distribution box and each distribution box are equipped with a classroom terminal controller PLC. The controller uses the Siemens s7-200 series as the control core, and consists of classroom power acquisition module, electrical switch control module, power module, and data communication module and other functional modules together to complete real-time collection of feature data within the classroom and dynamic control management. Classroom terminal controller PLC through the module comes with RS485 interface access 485 bus network, classroom terminal controller PLC data and PC management platform data and commands in the 485 bus network transmission, through the protocol conversion, and both data final get the corresponding control commands and data. Managers through the classroom terminal controller can view the use of various classroom resources, improve the efficiency of classroom resources, which can be beneficial to improve the comprehensive utilization of classroom resources and achieve classroom energy conservation and human management level

\section{Data acquisition Module}

Hall current sensor based on the magnetic balance Hall principle, the current Ic from the Hall element control current terminal, and in the normal direction of the Hall element applied magnetic field strength $\mathrm{B}$ of the magnetic field, Then in the direction perpendicular to the current and magnetic field (ie, between the Hall outputs) will produce a potential VH, called the Hall potential, its size is proportional to the control current I. According to this relationship can be collected by the sensor output voltage can reflect the size of the current. And the Hall effect of the Hall current sensor relative to other traditional current transformers have a higher accuracy and linearity, fast response time, bandwidth, anti-interference ability, overload and other advantages. In particular, the collection of small current sensor Hall has a greater advantage. Test results show that in the $220 \mathrm{~V}, 100 \mathrm{~W}$ classroom lighting test on the peak and peak of the sensor only 20mv change. ADC acquisition with the general method will produce relatively large error, and can not determine the amount of change, so the use of smooth mean filtering method to collect the data processing. First, the n-group data D $[1 \sim \mathrm{n}]$ are collected in one cycle, and then the data sequence is sorted from large to small and then the maximum value Dmax [i] and the minimum value Dmin [i] are obtained. Finally, Maximum and minimum data.The final peak-to-peak PP can be drawn:

$$
\mathrm{PP}=\sum_{i=1}^{S} D \max [i]-\sum_{i=1}^{s} D \min [i]
$$

Where the greater the accuracy of s higher, but there is a limiting factor is that when the $s$ increase the real-time efficiency of the data will be greatly reduced so according to the actual situation we use $\mathrm{s}=10$, which can ensure the accuracy of the situation but also to maintain the real-time data. 


\section{485 bus network communication mechanism}

RS-485 uses balanced transmission and differential reception, so it has the ability to suppress common mode interference. And coupled with the bus transceiver with high sensitivity, can detect as low as $200 \mathrm{mV}$ voltage, so the transmission signal can be recovered in kilometers away. RS- 485 halfduplex work, at any time only a little in the sending state. RS-485 is very convenient for multi-point interconnection, you can save a lot of signal lines. Application of RS-485 can be networked to form a distributed system. This system uses 485 bus to ensure the system's transmission rate and function requirements. In the communication protocol, the system uses the packet to send the header, address information, validity and data information contained in each packet. The specific communication flow is shown in Figure 2 below:

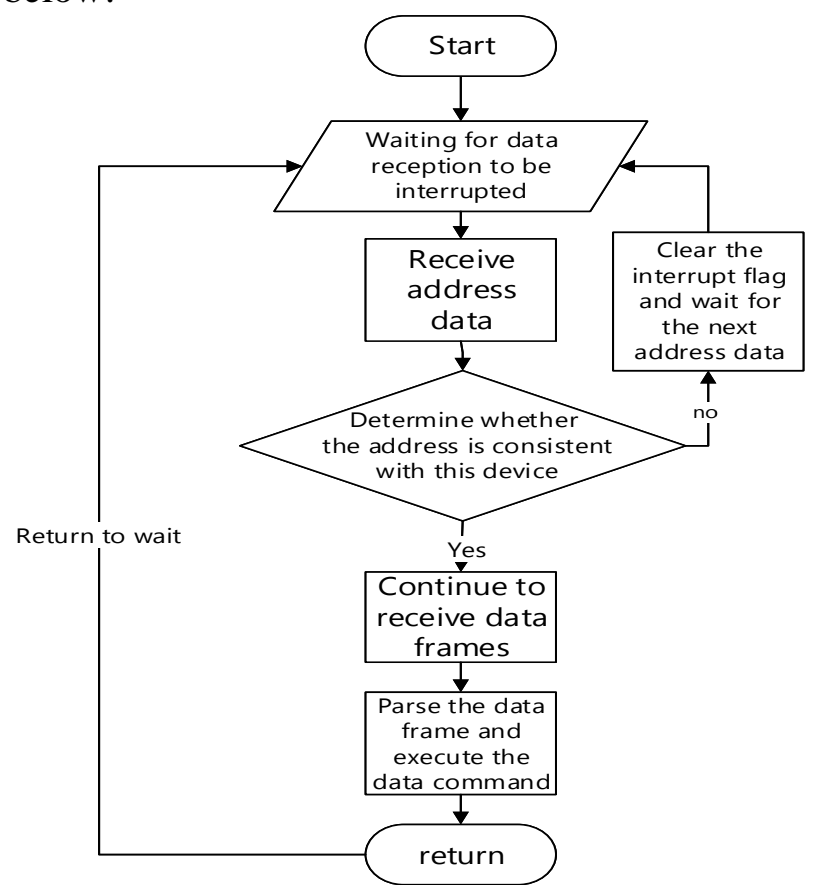

Figure 2. Flow chart

\section{Interpersonal interaction interface}

Computer is as the entire classroom resources energy-saving control system of the host computer to achieve the classroom display terminal resources, dynamic management and remote transmission of data information and other functions. Classroom resources energy-saving control system visualization of human-computer interface design interface shown in Figure 2:

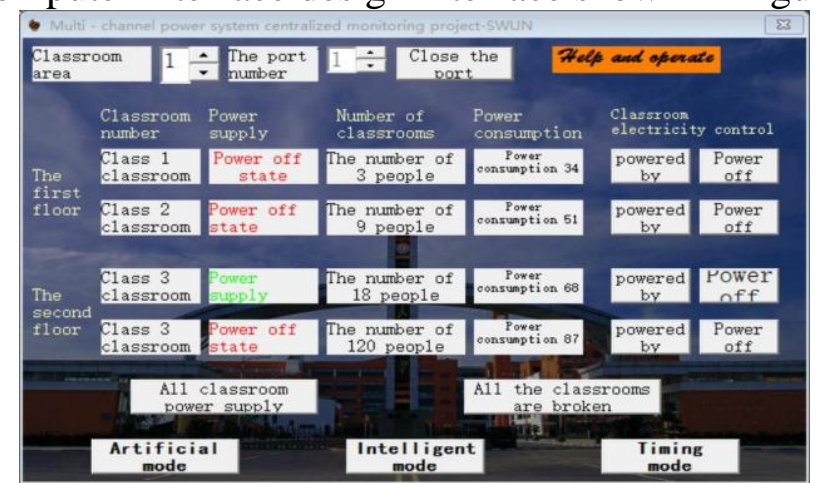

Figure 3. Classroom management platform interface

\section{Conclusion}

This paper mainly introduces the Multi - channel Electrical Information Acquisition and Control System Based on PLC and RS485 Bus Network. Using the reliability of the system, managers can 
use the system management platform to monitor the teaching building lighting, television and projectors and other electrical equipment in a distributed and distributed manner, so not only can save manpower and electricity costs, but also can compare the actual electricity consumption of electricity units, timely detection of failure, troubleshooting, with a good prospect.

\section{Acknowledgements}

This work was partially supported by National Nature Science Foundation (61673016), SWUN Construction Projects for Graduate Degree Programs (2017XWD-S0805), Advance Research Program of Electronic Science and Technology National Program (2017YYGZS16) and Sichuan Youth Science and technology innovation research team (2017TD0028), also was financially supported by students innovation training project of SWUN (No. S201610656069).

\section{References}

[1]Wei Yu. Design of classroom lighting adaptive control system based on CAN bus [J]. Technology horizon,2014,(35):190-191.

[2]Xin Jiang. Design of Multiple PLC Communication System Based on CAN Bus [J]. Electrical age,2011,(09):104-105.

[3]Zhaobo Han. Communication between Computer and PLC Based on CAN Bus [J]. Automation Technology \& Application,2009,(11):104-106.

[4]Zhenfeng Ma, Xianli Liu, Peng Wang, Xinmiao Jiang. Communication between PC and PLC Based on LabVIEW7.1 [J]. Journal of Harbin University of Science and Technology, 2005, (05):30-33+36.

[5]Xing Chen, Liquan Zhang, Wangwang Shi. Using VC ++ to Realize Serial Communication between PC and Multi - PLC [J]. Microcomputer information, 2004, (09):6-7+54.

[6]Chao Yu. Design and implementation of intelligent classroom management system based on RFID [D]. Jilin University, 2015.

[7]Shenghui Guo, Xueli Zhu, Shuxian Zhu. Classroom lighting system based on fuzzy control [J]. Journal of Illuminating Engineering, 2013,(03):127-130. 